\title{
HCI in the Era of Ambient Media - And beyond 2009 INTERACT Tutorial
}

\author{
Artur Lugmayr \\ Tampere University of Technology (TUT), POB. 553 \\ Korkeakoulunkatu 1, FIN-33101 Tampere, Finland \\ artur.lugmayr@tut.fi
}

\begin{abstract}
According to McLuhan, "the medium is the message" - but what means interaction and what is the medium in the age of ubiquitous and pervasive computation - when the medium is 'in' daily objects? Ambient media are media that are embedded throughout our natural environment - location based services, context awareness, emotional responsive interfaces, touch and gesture based interfaces, haptics and biometrics, sensor perception, mobile devices, and smart data mining are the technological enabler for smart media environments. The latest trends from emotional computation, affective computation, and tangible media lay the foundations for this new and exciting form of media existing far beyond screen concepts and mouse based interaction metaphors. The tutorial trains participants in the basic technologies as tools for the design of new interactive 'ambient' environments. It presents case studies and latest research results in the field of ambient media, ranging from ambient assisting living, user experience design, user contributed content, and mobile services. After the tutorial the participants should understand the principles of ambient media with its underlying concepts and methods, especially emphasizing humancomputer-interaction. As roundup, the tutorial presents a more visionary viewpoint to the future of media technology: the use of biological metaphors for interactive environments (biomedia).
\end{abstract}

Keywords: ubiquitous computation, ambient media, pervasive computation.

\section{Objectives and Schedule}

The goal of the tutorial is to train participants in the basics of ambient media especially focusing on the human-computer-interaction viewpoint. The tutorial is designed for a general audience with interest in a newly emerging media environment and its possibilities.

Monday Afternoon, $24^{\text {th }}$ August 2009

13:30-15:00 Part 1: Introduction, Concepts Overview, Technology

15:00-15:30 Coffee break

15:30-17:00 Part 2: Case Studies, Design Guidelines, Outlook in the Future

Please visit http://www.cs.tut.fi/ lartur for further material and information.

T. Gross et al. (Eds.): INTERACT 2009, Part II, LNCS 5727, pp. 938-939, 2009.

(C) IFIP International Federation for Information Processing 2009 


\section{Focus Points of the Tutorial}

The tutorial covers the following topics in further depth:

- case-studies of existing ambient media services

- basic concepts and technologies of ambient media

- location based services, mobile interaction, and smart environments

- user experience and interaction design guidelines

- ambient content production and creation

- natural and intuitive interaction methods

- context awareness and intelligent behavior modeling

- proactive and emotional responsive system designs

- ambient services and business models

- ambient social networks. 